\title{
High-Energy Astrophysics with Lobster Eye X-ray ASM
}

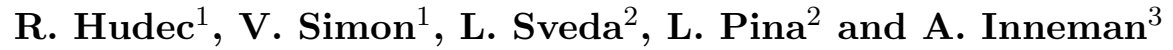 \\ ${ }^{1}$ Astronomical Institute, Academy of Sciences of the Czech Republic, CZ-251 65 Ondrejov, \\ Czech Republic \\ email: rhudec@asu.cas.cz \\ ${ }^{2}$ Czech Technical University, Faculty of Nuclear Physics, Prague, Czech Republic, \\ ${ }^{3}$ Center for Advanced X-ray Technologies, Reflex sro, Prague, Czech Republic
}

\begin{abstract}
We report on astrophysical aspects of fully innovative very wide-field X-ray telescopes with high sensitivity. They are expected to contribute essentially to study of various astrophysical objects such as AGN, SNe, Gamma-ray bursts (GRBs), X-ray flashes (XRFs), galactic binary sources, stars, CVs, X-ray novae, various transient sources, etc.
\end{abstract}

Keywords. X-ray telescopes, All-Sky Monitor

\section{Introduction}

The X-ray sky is highly variable, rich in variable and transient sources of both galactic as well as extragalactic origin. However, since many of these transient events cannot be predicted, and are relatively rare, very wide-field instruments are required. They must achieve high sensitivities and provide precise localizations in order to effectively study the objects. Wide field X-ray telescopes with imaging optics are expected to represent an important tool in future space astronomy projects in general, especially those for deep monitoring and surveys in X-rays over a wide energy range. The Lobster-Eye (LE) wide field X-ray optics has been suggested in 70ies by Schmidt (Schmidt, 1975, orthogonal stacks of reflectors) and by Angel (Angel, 1979, array of square cells). Up to 180 deg FOV may be achieved. This novel X-ray optics offers an excellent opportunity to achieve very wide fields of view (FOV, 1000 square degrees and more) while the widely used classical Wolter grazing incidence mirrors are limited to roughly 1 deg FOV (Priedhorsky et al., 1996, Inneman et al., 2000).

\section{Science}

Deep (limiting flux of $10^{-12} \mathrm{erg} \mathrm{cm}^{-2} \mathrm{~s}^{-1}$ can be easily achieved for daily scanning observation) X-ray sky monitoring with large FOVs (e.g. FOV of $6 \times 180$ deg can be easily assembled on the space station ISS) is expected to contribute significantly to various fields of modern astrophysics. A few most important examples are listed below.

(1) Gamma Ray Bursts (GRBs). Detection rates of nearly 20 GRBs/year can be obtained for the prompt X-ray emission of GRBs, taking into account the expected GRB rate $300 /$ year. (2) X-ray flashes. Detection rates of nearly $8 \mathrm{X}$-ray flashes/year are expected, assuming XRF rate of 100/year. (3) X-ray binaries. Because of their high variability in X-rays they will be one of major targets in LE observations. LE will be able to observe their short-time outbursts by long-term extended monitoring. Almost all galactic $\mathrm{XRB}$ are expected to be within the detection limits. (4) Stars. Because of the low X-ray luminosity of ordinary stars, only nearby stars are expected to be observable. We estimate the lower limit of ordinary stars observable by the LE telescope as 600 . The sampling 
rate of LE observations will be sufficient enough to observe sudden X-ray flux increases during flares while still having the capability of monitoring the variability on time scales of years.(5) Supernovae. The LE telescope should be able to detect the theoretically predicted thermal flash lasting for $\sim 1000 \mathrm{sec}$ for the first time. Together with the optical SNe detection rate and estimates of the LE FOV we estimate the total number of SNe thermal flashes observed by the LE experiment to 10/year. (6) AGNs. Active Galactic Nuclei will surely be one of the key targets of the LE experiment. LE will be able to monitor the behavior of the large $(\sim 1000)$ sample of AGNs providing long-term observational data with good time sampling (hours). (7) X-ray transients. The LE experiment will be ideal to observe X-ray transients of various nature due to its ability to observe the whole sky several times a day for a long time with a limiting flux of about $10^{-12} \mathrm{erg} \mathrm{cm}^{-2} \mathrm{~s}^{-1}$. More and fainter X-ray transients are expected to be detected by the LE sky monitor enabling the detailed study of these phenomena. (8) Cataclysmic Variables. Cataclysmic Variables $(\mathrm{CVs})$ are very active galactic objects, often showing violent long-term activity in both the optical and X-ray passband (outbursts, high/low state transitions, nova explosions) as well as rapid transitions between the states of activity. Search for the relation of the optical and X-ray activity is very important - monitoring of a large number of CVs is necessary to catch them in various states of activity. Most up to now X-ray observations of CVs: (i) Snapshots catching selected CVs in a particular state of activity, (ii) In most cases the transitions between the states are not covered, and (iii) Poor statistics of phenomena and objects (deeper studies available for only a few CVs). Important classes of CVs for LOBSTER are Non-magnetic dwarf novae (DNe), Supersoft X-ray sources (SSXSs), Classical novae (CNe), and Polars with soft X-ray excess.

\section{Conclusions}

Analysis and simulations of Lobster-Eye X-ray telescopes have been carried out. They have indicated that these innovative devices will be able to monitor the X-ray sky at an unprecedented level of sensitivity, an order of magnitude better than any previous X-ray all-sky monitor. Limits as faint as $10^{-12} \mathrm{erg} \mathrm{cm}^{-2} \mathrm{~s}^{-1}$ for daily scanning observation as well as the angular resolution $<4$ arcmin in soft X-ray range are expected to be achieved allowing monitoring of all classes of X-ray sources, not only X-ray binaries, but also fainter classes such as AGNs, coronal sources, cataclysmic variables, as well as fast X-ray transients including gamma-ray bursts and the nearby type II supernovae. The various prototypes of both Schmidt as well as Angel arrangements have been produced and tested successfully, demonstrating the possibility to construct these lenses by innovative but feasible technologies. Both very small Schmidt lenses $(3 \times 3 \mathrm{~mm})$ as well as large lenses $(300 \times 300 \mathrm{~mm})$ have been developed, constructed, and tested. This makes the proposals for space projects with very wide field lobster eye optics possible for the first time.

\section{Acknowledgements}

We acknowledge the support provided by the Ministry of Industry and Trade of the Czech Republic, FD-K3/052, and partly by the Grant Agency of the AS CR (A3003206) and Grant Agency of the Czech Rep. (205/05/2167).

\section{References}

Angel J. R. P., 1979, ApJ, 364, 233

Inneman A. et al., 2000, Proc. SPIE, 4138, 94

Priedhorsky, W. C. et al., 1996, MNRAS 279, 733

Schmidt, W. K. H., 1975, NucIM, 127, 285 